\title{
Editorial
}

\section{Complex Differences and Difference Equations}

\author{
Zong-Xuan Chen, ${ }^{1}$ Kwang Ho Shon, ${ }^{2}$ and Zhi-Bo Huang ${ }^{1}$ \\ ${ }^{1}$ School of Mathematical Sciences, South China Normal University, Guangzhou 510631, China \\ ${ }^{2}$ Department of Mathematics, Pusan National University, Busan 609-735, Republic of Korea
}

Correspondence should be addressed to Zong-Xuan Chen; chzx@vip.sina.com

Received 12 August 2014; Accepted 12 August 2014; Published 22 December 2014

Copyright (c) 2014 Zong-Xuan Chen et al. This is an open access article distributed under the Creative Commons Attribution License, which permits unrestricted use, distribution, and reproduction in any medium, provided the original work is properly cited.

In more recent years, activity in the area of the complex differences and the complex difference equations has fleetly increased.

This journal has set up a column of this special issue. We were pleased to invite the interested authors to contribute their original research papers as well as good expository papers to this special issue that will make better improvement on the theory of complex differences and difference equations.

In this special issue, many good results are obtained.

Difference equations are widely applied to mathematical physics, economics, and chemistry. In this special issue, Z.B. Huang and R.-R. Zhang, J. Li et al., and L. Gao and Y. Wang investigate the growth, a Borel exceptional value of meromorphic solutions to different types of higher order nonliear difference equations, respectively; B. Chen and S. Li investigate the Schwarzian type difference equation. D. Liu et al., Z. Mao and H. Liu, and S. Li and B. Chen investigate unicity of meromorphic functions concerning different types of difference operators. Recently, many difference analogues of the classic Nevanlinna theory are obtained.

In this special issue, X.-M. Zheng and H. Y. Xu obtain a differential difference analogue of Valiron-Mohonko theorem. Related topics with complex difference, J. E. Kim and K. H. Shon investigate the regularity of functions on dual split quaternions in Clifford analysis and the tensor product representation of polynomials of weak type in a DF-space; Q. Zhang and Z. Liu et al. investigate different types of real difference equations, respectively; L. Shen and Q. Xu investigate stochastic differential equations.
This special issue stimulates the continuing efforts to the complex differences and the complex difference equations.

$$
\begin{array}{r}
\text { Zong-Xuan Chen } \\
\text { Kwang Ho Shon } \\
\text { Zhi-Bo Huang }
\end{array}
$$




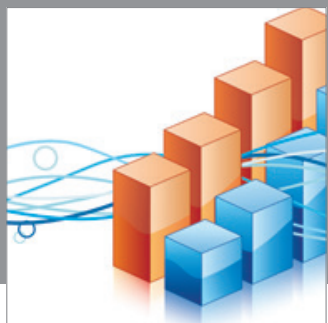

Advances in

Operations Research

mansans

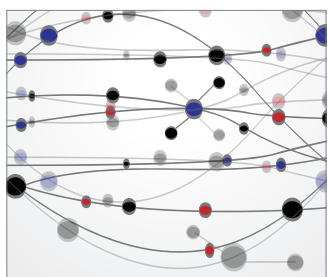

The Scientific World Journal
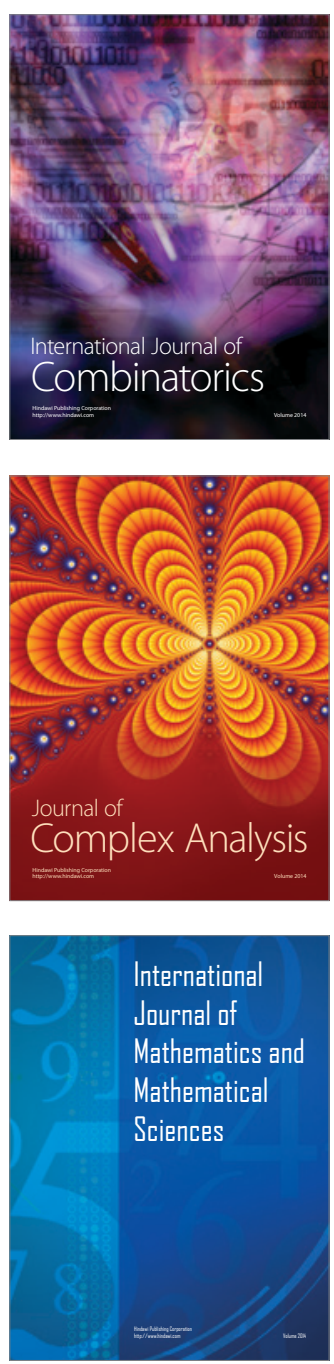
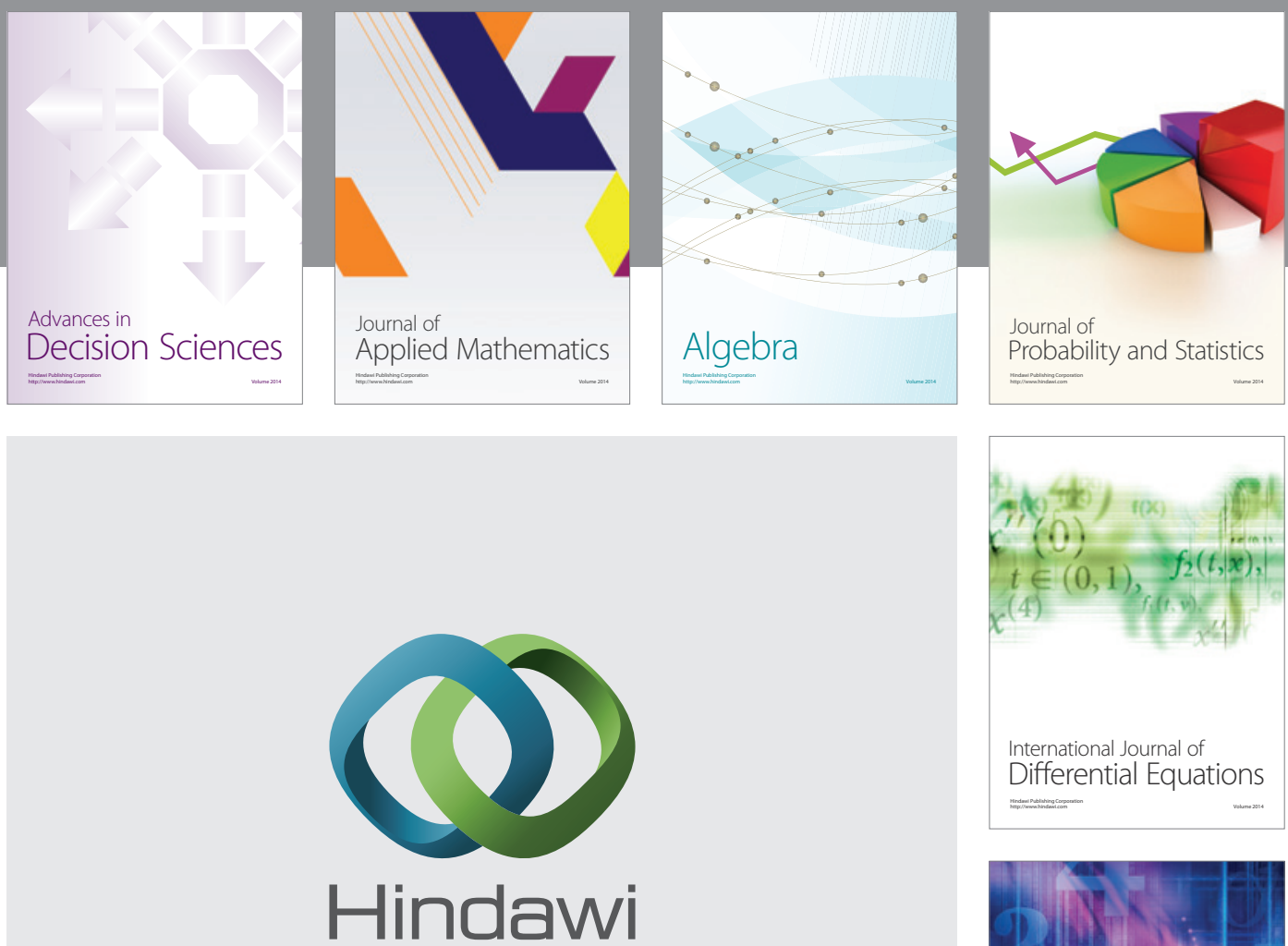

Submit your manuscripts at http://www.hindawi.com
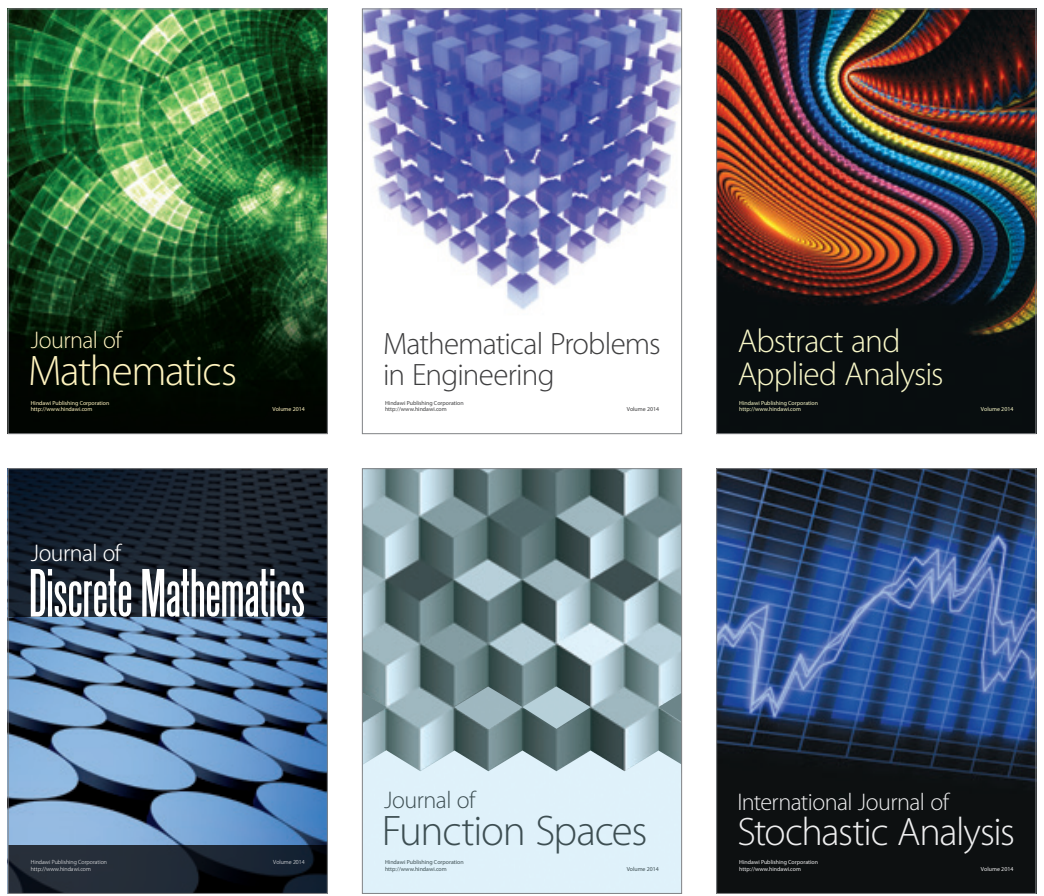

Journal of

Function Spaces

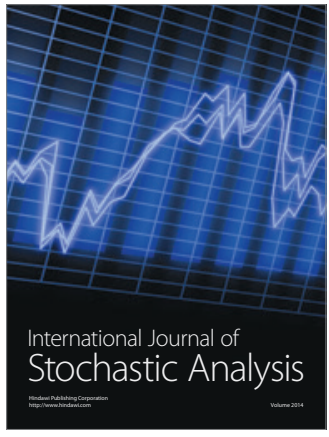

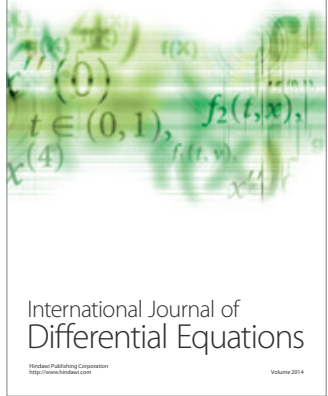
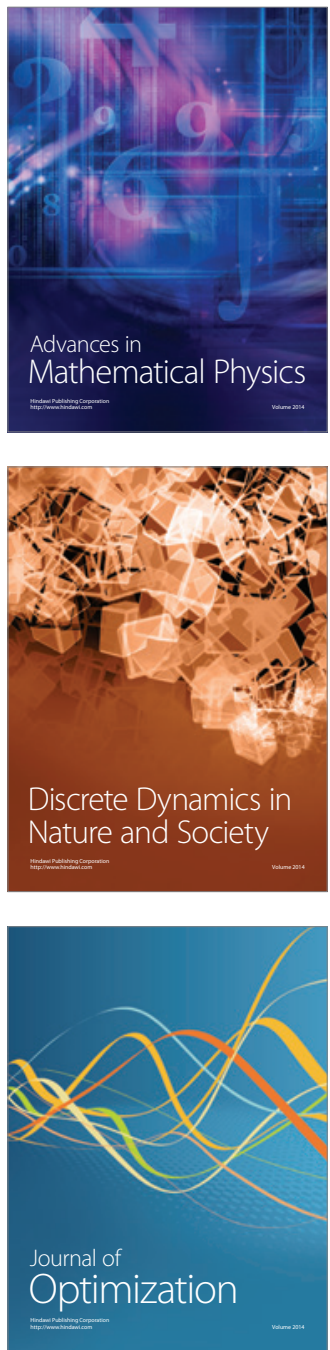